This paper is published in the open archive of Mid Sweden University

DIVA http://miun.diva-portal.org

with permission of the publisher

Citation for the peer-reviewed published paper:

Yang L, Fogden A, Pauler N, Sävborg Ö, Kruse B. A novel method for studying ink penetration of a print. Nordic Pulp \& Paper Research Journal. $2005 ; 20(4): 423-429$.

URL to article at publishers site:

http://dx.doi.org/10.3183/NPPRJ-2005-20-04-p423-429 


\title{
A novel method for studying ink penetration of a print
}

\author{
Li Yang, Karlstad University, Karlstad, Sweden, Andrew Fogden, Institute for Surface Chemistry, YKI, Stockholm, Sweden, Nils Pauler, Mid Sweden \\ University, Örnsköldsvik, Sweden, Örjan Sävborg, Stora Enso Research Center, Falun, Sweden, Björn Kruse, Linköping University, Norrköping, Sweden
}

KEYWORDS: Ink penetration, Image processing, Ink paper interaction

SUMMARY: A method combining microscopy with image analysis for studying ink penetration of ink-jet prints has been proposed. Prints on paper substrates of various material compositions have been microtomed, imaged with optical microscopy, and analyzed with image processing. The penetration depth and distribution of the ink-jet dye in the paper, which depend on the material compositions of the substrates and printed ink volumes, are then obtained by means of statistical analysis. The study focuses on uncoated fine papers, including five types of hand-sheets and two commercial papers. It is observed that ink penetration can be significantly reduced by adding internal sizing (hydrophobizing) chemicals in the hand-sheet making, while it is relatively less affected by addition of filler and fluorescent whitening agent (FWA). Ink volume obviously influences the depth of its penetration, with depth increasing linearly for higher ink volumes but displaying a disproportionately shallow penetration for smaller applied volumes.

ADDRESSES OF THE AUTHORS: Li Yang: Karlstad University, Department of Chemical Engineering, SE-651 88 Karlstad, Sweden. Andrew Fogden: Institute for Surface Chemistry, YKI, SE-114 28 Stockholm, Sweden. Nils Pauler: Mid Sweden University, Digital Printing Centre (DPC), SE-891 18 Örnsköldsvik, Sweden. Örjan Sävborg: Stora Enso Falun Research Center, SE-791 80 Falun, Sweden. Björn Kruse: Linköping University, Department of Science and Technology, 60174 Norrköping, Sweden.

Corresponding author: Li Yang (li.yang@kau.se)

An ink-jet printing system consists of three fundamental parts: inks, printing engine, and substrate. Inks are materials creating colour by selectively absorbing and scattering the visible illumination light. The printer acts as an ink distributor that governs the ink application. Correspondingly, the substrate acts as a receiver of the inks and forms the images. Ink setting on the substrate is a complex process that depends on physical and chemical properties of the inks and the substrates, and their interactions. Absorption of ink constituents by the substrate, or ink penetration, is significant over a range of timescales, from the first stages of transfer and drying by imbibition, diffusion and evaporation, through to longterm stability (Voet 1952, Mizutani, Kondo 2000, Rousu 2002). For a system consisting of dye-based liquid inks and plain paper, the dyes move together with the liquid carrier until the combination of vehicle evaporation and absorption into pores immobilizes the dye. This process can contribute to serious ink spreading on the surface along the paper fibres ("wicking"). At the same time, the ink spreads downwards within the internal pore structure. This can cause severe dot deformation (Emmel, Hersch 2002), physical dot gain, and ink strike-through.
Understanding the consequences of these phenomena and, above all, being able to quantitatively characterize the ink penetration and its impact on colour reproduction, is of great importance.

Ink penetration has long been an important topic in offset printing, where the separation of components during ink setting and drying affects a wide range of printability and print quality issues, such as trapping and rub-off, print gloss and density, print mottle and print through, etc (Ström et al 2000, Rousu 2002). A variety of microscopic techniques, having the potential for measuring penetration of ink components into print media, have been developed, for instance, SEM (Scanning electron microscopy) of cross-sections of a corrugated board (Fayyazi 2002), SWLI (Scanning white-light interferometric) measurements of surface topography (McKnight et al 2001) CLSM (Confocal laser scanning microscopy, Sung et al 2002) for depth imaging, etc. Other techniques include various forms of spectroscopy and combinations with microscopy, e.g. Confocal Raman microscopy (Vyörykkä 2004). Broadly speaking, most spectroscopic techniques, although having the potential of giving more information and with less sample preparation, have been found to be difficult to directly interpret, often requiring substantial calibration procedures and support from more direct techniques. The well-established SEM procedures (Kishida et al 2001) for analyzing local ink-film thickness of offset prints are of little use for studying ink penetration of ink-jet prints, because of the low electrondensity contrast of the colorants and lack of a clearly defined ink film. Instead, optical microscopy will be the main tool in the present study, as it provides direct colorant information with a satisfactory resolution of around $1 \mu \mathrm{m}$.

On the theoretical side, Bristow (Bristow 1987) and Pauler (Pauler 1987) proposed a method based on Kubelka-Munk (K-M) theory (Kubelka, Munk 1931, Kubelka 1954) for estimating the depth of ink penetration from spectral reflectance measurements. One limitation of this method is the assumption of homogeneous ink distribution in the substrate within a constant depth of ink penetration. Thus, only a simplistic characterization in the form of an average depth of ink penetration can be obtained, and more detailed information regarding the true form and density of the ink distribution is not available. For inhomogeneous ink penetration, theoretical extension of the K-M theory has to be made, as recently suggested (Yang, Kruse 2000, Yang et al 2004). In the extended theory, pre-knowledge about the form of the ink distribution in the substrate is essential.

Quantitative characterization of ink penetration, i.e. forms (homogeneous or otherwise) and depths and their dependence on properties of inks and paper, have largely 
remained unsolved problems in ink-jet printing. Therefore, this work aims at establishing the methodologies, by combining optical microscopy with image analysis, to gain insight into the characteristics of ink penetration, and in particular, ink density distribution. The results provide, to the best of our knowledge, the first information on ink-jet ink penetration to this high level of resolution and quantification.

\section{Methodology}

The study addressed uncoated fine paper and comprised five types of hand-sheets plus two commercial office copy papers, all of grammage $80 \mathrm{~g} / \mathrm{m}^{2}$. The hand-sheets were specially fabricated at MoRe Research using the Formette Dynamique. They consisted of a mixture of $60 \%$ birch $\left(24{ }^{\circ} \mathrm{SR}\right)$ and $40 \%$ pine $\left(19{ }^{\circ} \mathrm{SR}\right) \mathrm{kraft}$ pulp fibres (abbreviated $\mathrm{W}$ ), with or without calcium carbonate filler (20\% PCC, abbreviated F), and internal size (Approximately $0.3 \%$ ASA, abbreviated S), and together with typical dry strength, drainage and retention additives. By mistake, three of the five types of hand-sheets contained fluorescent whitening agents (FWA, abbreviated X). The compositions of these hand-sheets can thus be abbreviated as, for example, $\mathrm{W}_{1} \mathrm{~F}_{1} \mathrm{~S}_{0} \mathrm{X}_{1}$ and $\mathrm{W}_{1} \mathrm{~F}_{0} \mathrm{~S}_{1} \mathrm{X}_{0}$, representing hand-sheets consisting of pulp $\left(\mathrm{W}_{1}\right)$ and filler $\left(\mathrm{F}_{1}\right)$ with FWA $\left(\mathrm{X}_{1}\right)$, and pulp $\left(\mathrm{W}_{1}\right)$ and internal size $\left(\mathrm{S}_{1}\right)$, respectively. The type and amount of filler, type of internal size and Cobb value thus obtained, and additives, were all chosen to be similar to those of the commercial samples, thus hand-sheet $\mathrm{W}_{1} \mathrm{~F}_{1} \mathrm{~S}_{1} \mathrm{X}_{1}$ is most similar to the commercial papers. However, none of the hand-sheets were surface sized, in distinction to the commercial samples. The printing was performed with a Desktop HP 970 printer that controls the ink level in six steps. The printed ink amount (number of ink droplets fired) was controlled in such a way, through home-developed software based on PCL (printer command language), so as to be proportional to the ink levels. In a former investigation it was shown that the ink amount increases linearly with the ink level, varying from approximately 0.8 to $5.2 \mathrm{~g} / \mathrm{m}^{2}$ (Pauler et al 2002). Full tone colour patches were printed on each paper with primary $(\mathrm{C}, \mathrm{M}, \mathrm{Y})$ and secondary $(\mathrm{R}$, $\mathrm{G}, \mathrm{B})$ colours, i.e. only involving the dye-based inks and not the pigment-based black $(\mathrm{K})$.

\section{Micro-imaging}

A $7 \times 15 \mathrm{~mm}$ piece within each printed patch was mounted, vacuum impregnated with liquid methacrylate plastic, heat-cured, and trimmed to remove excess plastic in the thickness-direction. The embedded sample was then microtomed with a diamond knife to a thickness of approximately $3 \mu \mathrm{m}$, with two separate cuts performed for each sample. Both the cutting and transferring of the microtomes to the optical microscope were performed in the dry state. The avoidance of water in the procedure, together with the use of an appropriate embedding plastic, ensured that the distribution of the printed dye was not altered during the preparation. The optical microscope, using a Nikon digital camera with ACT-1 camera software, was used at around 20x magnification to obtain a series of images taken with small adjustments of the focus (necessary since the thin microtomed sample typically does not lie totally flat). These images were combined using Nikon Easy Image Analysis to provide one complete image in full focus, then stored in files of Jpeg format.

Each original image has a size of $1280 \times 1024 \times 3$, corresponding to the width (pixel) and height (pixel) of the image, and three colour channels, respectively, with the height in the direction of paper thickness. The height is reduced to $400-500$ (pixels) after removing a large portion of background (the embedding polymer) from the image.

\section{Image processing}

Fig 1 is a schematic diagram of the cross section of a print, in which the areas I and IV correspond to the embedding material and the areas II and III to the inked and non-inked portions of the paper sheet, respectively. Due to local inhomogeneity in paper structure, the depth of ink penetration varies significantly from one image column to another (as do the paper surfaces and their local thickness, represented in Fig 1 as straight line for simplicity).

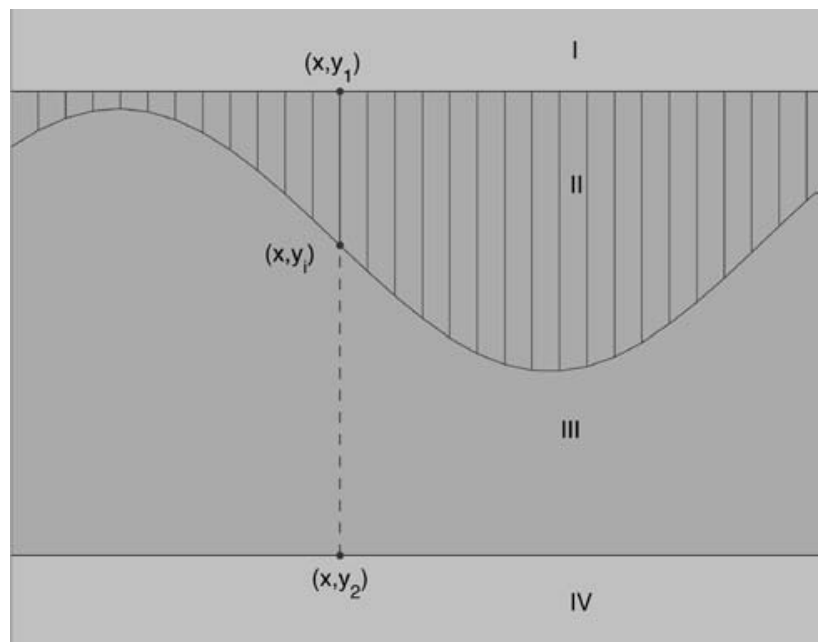

Fig 1. A schematic diagram of ink penetration. The cross section consists of four sub-areas, with I and IV being the layers of embedding material, and II and III being the inked and clean portions of the paper sheets, respectively.

The aim of image processing is to separate the layer of a paper sheet from the embedding material (image background) and to distinguish the border of ink penetration. The main technique used is intensity thresholding, discriminating an image area from another. As an image consists of intensity matrices of the three imaging channels, red, green, and blue, making use of information from all these channels can greatly simplify the thresholding processes. Fig $2 a$ ) is a typical example of intensity variations (in different colour channels) along an image column. Clearly, the image pixels in the image column can be divided into four groups. Groups $A$ and $D$ correspond to the embedding material, $B$ the paper sheet with ink penetration, and $\mathrm{C}$ the remaining (non-inked) part of the paper sheet.

For simplicity, we use a print of cyan as example to explain our method for image analysis. Extensions to 

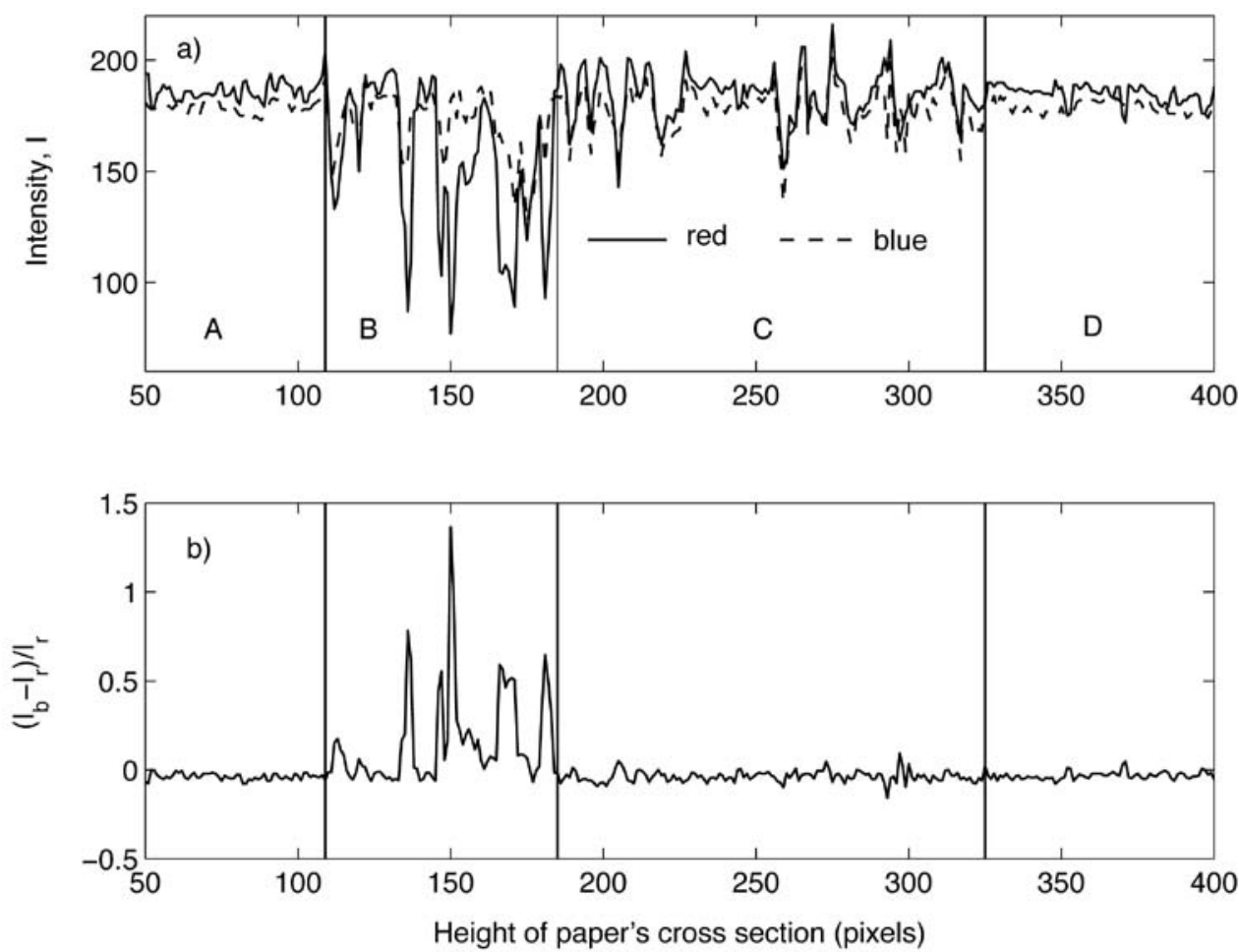

Fig 2. An example of intensity variations in different colour channels along an image column, for a cyan print (ink level 3 ) on the hand-sheet, $W_{1} F_{1} S_{0} X_{1},{ }_{0}$ consisting of fibres and fillers. a) Intensity variations in red and blue imaging channels. b) The relative intensity variation computed with Eq. (1).

prints of other colours are straightforward. Because the image area with cyan ink has weaker intensity in the red channel compared to that in blue, the red channel is therefore a proper candidate for image thresholding, separating the top surface of a printed paper sheet from the light grey background (group A) of the embedding.

The different intensities in different image channels are particularly useful information when separating the portion with ink penetration from the remaining part of the paper sheet. As fillers in paper sheets have (darker) grey tone, which may cause difficulties in discriminating inks from fillers, if only one image channel is used. This problem can be solved by using an extra image channel. For example, cyan ink has weak intensity in the red channel, $\mathrm{I}_{\mathrm{r}}(\mathrm{x}, \mathrm{y})$, but strong intensity in the blue, $\mathrm{I}_{\mathrm{b}}(\mathrm{x}, \mathrm{y})$, with $\mathrm{x}, \mathrm{y}$ denoting a column and a row in the image. A combination of these two channels, i.e.,

$$
\frac{I_{b}(x, y)-I_{r}(x, y)}{I_{r}(x, y)} \geq \varepsilon,
$$

can be a proper indicator that discriminates a colour pixel from a grey, as seen from Fig $2 b$. The quantity $\varepsilon$ in $E q$ (1) is a criterion for thresholding, whose value varies from colour to colour and even from image to image because of inconsistency of image backgrounds.

Similar descriptors to $E q$ (1) have been applied successfully to prints of other primary colours as well as secondary colours. The lower surface of a paper sheet is determined by thresholding using grey images.

\section{Statistical analysis}

Image processing that classifies interfaces between the embedding layers and paper sheet and between the portion of paper with and without ink penetration, forms the ground for image analysis. The image thresholding is done column-wise. As image intensities vary dramatically from column to column, statistical treatments of image data obtained from the image analysis are needed.

Let $\mathrm{y}_{1}(\mathrm{x})$ and $\mathrm{y}_{2}(\mathrm{x})\left(\mathrm{y}_{2}>\mathrm{y}_{1}\right)$ be the rows (heights) of upper and lower surfaces of a paper sheet (see Fig 1), respectively, with $x$ denoting an image column. We further assume that the interface between the portion with ink penetration and the rest of paper sheet lies at $\mathrm{y}_{\mathrm{i}}(\mathrm{x})$. One can then compute the ink penetration (in percentage) along the column by taking the ratio of the local thickness of ink penetration to the local thickness of paper sheet, i.e.,

$$
d_{i}(x)=\frac{y_{i}(x)-y_{1}(x)}{y_{2}(x)-y_{1}(x)} \times 100 \% .
$$

The mean depth of ink penetration can then be computed by averaging over all image columns, i.e.,

$$
\bar{d}_{i}=\left[\sum_{x=1}^{1280} d_{i}(x)\right] / 1280,
$$

which is comparable to the theoretical computations based on spectroscopic measurements (Bristow 1987, Pauler 1987).

To characterize the distribution of ink penetration in a way that is more statistically meaningful, we further introduce two quantities, Pop and Den, representing population of depth of ink penetration and density of ink penetration, respectively. The quantity, Pop, is defined as the histogram that bins $\mathrm{d}_{\mathrm{i}}(\mathrm{x})$ into 100 equally spaced 
intervals of the paper thickness, i.e.

$$
\operatorname{Pop}(m)=\operatorname{HIST}\left(d_{i}(x), 100\right), \quad(m=1,2, \ldots, 100)
$$

From the histogram distribution, one can obtain an even more collective descriptor of ink penetration, Den, the density of ink penetration, which is defined as

$$
\operatorname{Den}(n)=\sum_{m=n}^{100} \operatorname{Pop}(m), \quad(n=1,2, \ldots, 100)
$$

The quantities defined above, describing characteristics of ink penetration, depend naturally on paper compositions (fibres and additives), ink compositions (colorants

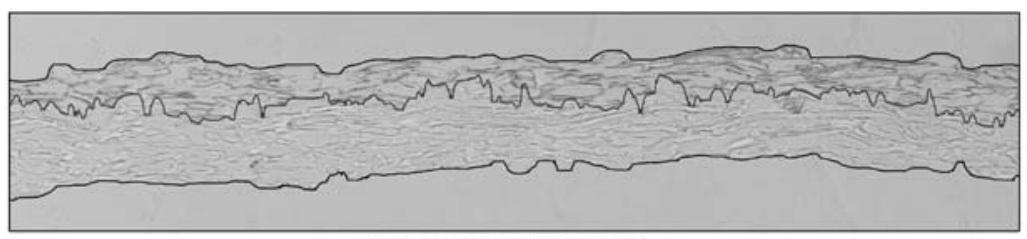

(a) substrate: $\mathrm{W}_{1} \mathrm{~F}_{0} \mathrm{~S}_{0} \mathrm{X}_{0}$

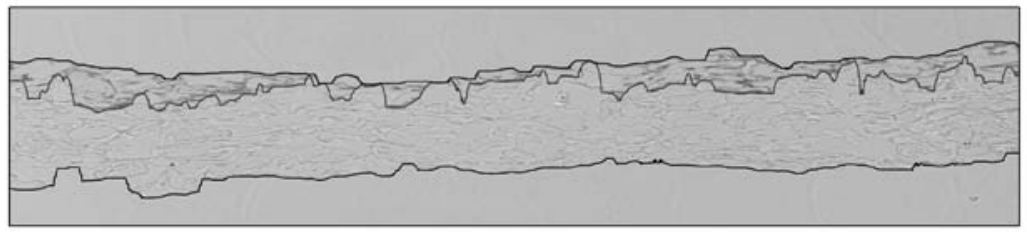

(b) substrate: $\mathrm{W}_{1} \mathrm{~F}_{0} \mathrm{~S}_{1} \mathrm{X}_{0}$

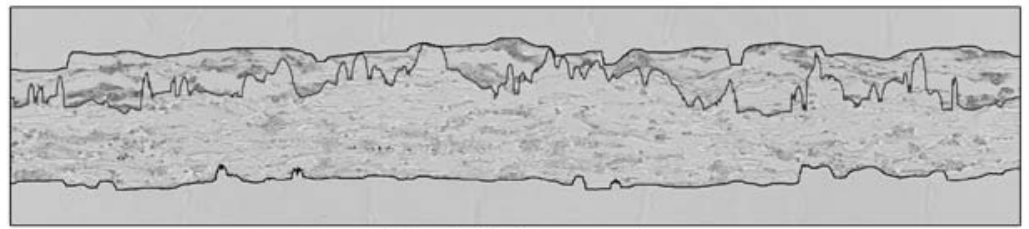

(c) substrate: paper 1

Fig 3. Image classifications for top and bottom surfaces of paper sheets as well as interfaces of ink penetration. The samples are printed with (cyan) ink level 3 and on hand-sheets $W_{1} F_{0} S_{0} X_{1}, W_{1} F_{0} S_{0} X_{1}$, and office copy paper (paper sample 1), respectively.

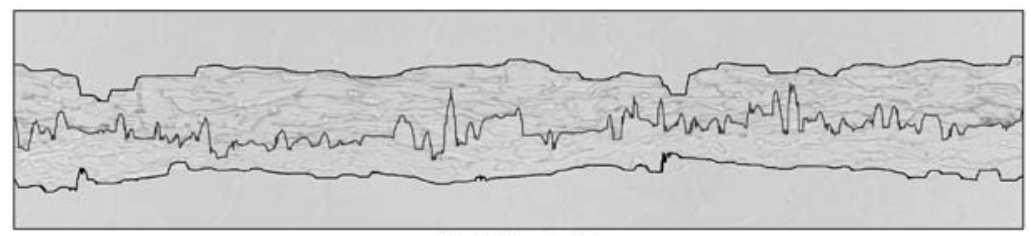

(a) $\mathrm{W}_{1} \mathrm{~F}_{0} \mathrm{~S}_{0} \mathrm{X}_{1}$

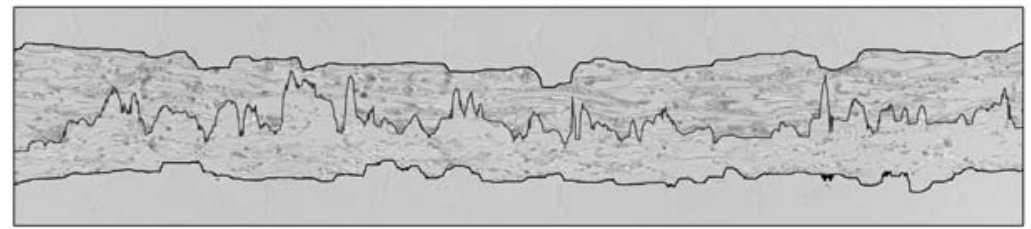

(b) $W_{1} F_{1} S_{0} X$

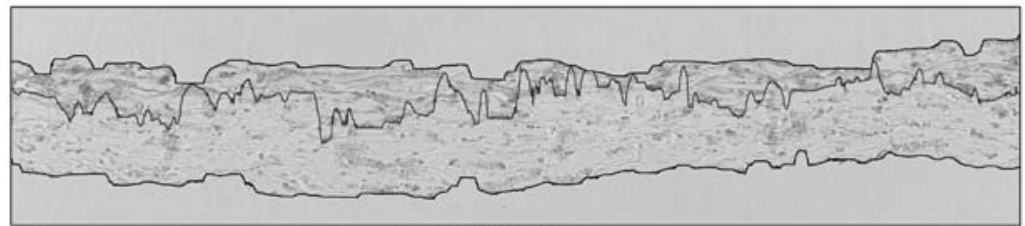

(c) $\mathrm{W}_{1} \mathrm{~F}_{1} \mathrm{~S}_{1} \mathrm{X}_{1}$

Fig 4. Dependence of ink penetration on compositions of papermaking (fillers, sizing materials, etc.). Three types of hand-sheets, $W_{1} F_{0} S_{0} X_{1}$ (without filler), $W_{1} F_{1} S_{0} X_{1}$ (with filler), and $W_{1} F_{1} S_{1} X_{1}$ (with filler and internal size), are printed with green ink and ink level 3 . and solutions), and even printing ink amount, as one will

\section{Results and Discussions}

mage processing and statistical analysis have been conducted for images of prints with primary and secondary sition and two commercial office copy papers (labelled mples 1 and 2).

ig 3 depicts the layer classifications for prints with the same colour (cyan) and printing ink level (3) but on different types of substrate $\left(\mathrm{W}_{1} \mathrm{~F}_{0} \mathrm{~S}_{0} \mathrm{X}_{0}, \mathrm{~W}_{1} \mathrm{~F}_{0} \mathrm{~S}_{1} \mathrm{X}_{0}\right.$, and the commercial sample 1). The top and bottom surfaces of the paper sheets are denoted by lines and the interface of ink penetration by the line inbetween. The ink is clearly rather uniformly distributed around fibres in $\mathrm{W}_{1} \mathrm{~F}_{0} \mathrm{~S}_{0} \mathrm{X}_{0}$, without filler or internal sizie chemical. On addition of internal size, $\mathrm{W}_{1} \mathrm{~F}_{0} \mathrm{~S}_{1} \mathrm{X}_{0}$, the depth of ink penetration is significantly reduced, as would be expected from the hydrophobization of the fibres. At the same time the dye distribution is less homogeneous, microscopically, due to the more limited pathways for liquid absorption and possibly to inhomogeneous distribution of the sizing chemical. This contributes to the prints on $\mathrm{W}_{1} \mathrm{~F}_{0} \mathrm{~S}_{1} \mathrm{X}_{0}$ having higher colour saturation and correspondingly less print through than on $\mathrm{W}_{1} \mathrm{~F}_{0} \mathrm{~S}_{0} \mathrm{X}_{0}$. The commercial paper sample 1, containing pulp, filler, internal and surface size etc., shares some similarity to that of $\mathrm{W}_{1} \mathrm{~F}_{0} \mathrm{~S}_{1} \mathrm{X}_{0}$ but with a larger depth of ink penetration. Further examinations of prints with different colours and different printing ink amounts have confirmed these general observations.

The filler used in these papers significantly increases the brightness and opacity of the sheets. Its effect on ink penetration is, however, rather limited as suggested by Fig 4 (note that the depth of ink penetration should be compared to the thickness of the paper), with comparisons between $\mathrm{W}_{1} \mathrm{~F}_{1} \mathrm{~S}_{0} \mathrm{X}_{1}$ (with filler) and $\mathrm{W}_{1} \mathrm{~F}_{0} \mathrm{~S}_{0} \mathrm{X}_{1}$ (no filler). The effect of internal sizing, $\mathrm{W}_{1} \mathrm{~F}_{1} \mathrm{~S}_{1} \mathrm{X}_{1}$, on the other hand, is significant, as observed previously.

Figs 3 and 4 demonstrate a strong dependence of ink penetration on the paper's composition. They show also dramatic variation in depth of ink penetration from column to column in the image matrix, resulting from local inhomogeneity in paper structure (pores, filler particle clusters, etc.).

Fig 5 depicts the histogram population, $P o p$, in respect to depth of ink penetration of cyan (level 3) on substrates $\mathrm{W}_{1} \mathrm{~F}_{0} \mathrm{~S}_{0} \mathrm{X}_{0}$, $\mathrm{W}_{1} \mathrm{~F}_{0} \mathrm{~S}_{1} \mathrm{X}_{0}$, and commercial sample 1. The 


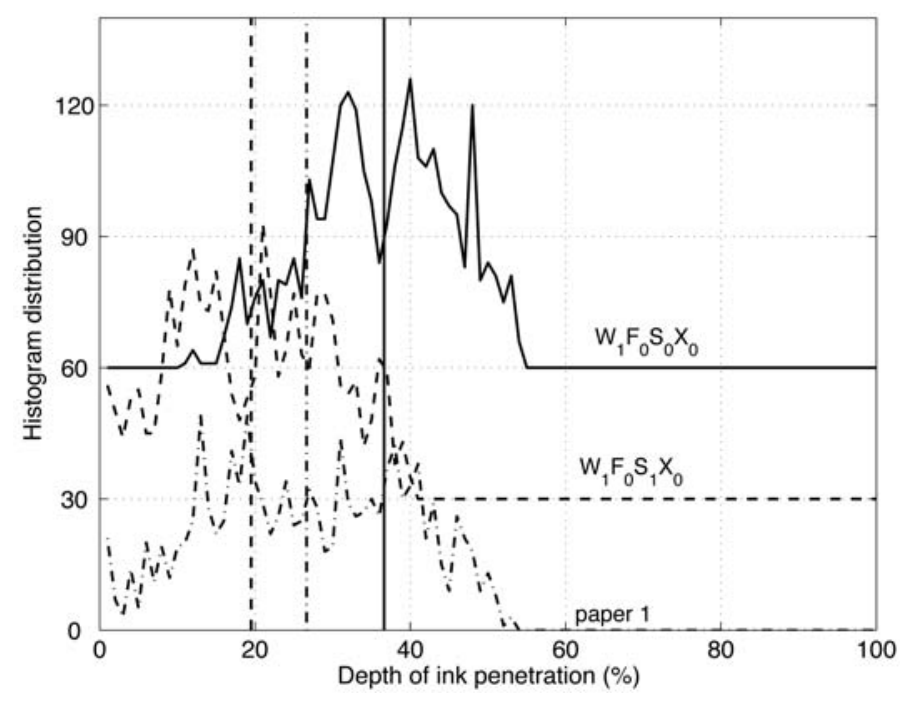

Fig 5. The histogram distribution (population) of depth of ink-penetration. The samples are printed with cyan (ink level 3) on $\mathrm{W}_{1} \mathrm{~F}_{0} \mathrm{~S}_{0} \mathrm{X}_{0}, \mathrm{~W}_{1} \mathrm{~F}_{0} \mathrm{~S}_{1} \mathrm{X}_{0}$, and commercial paper (sample 1). The vertical lines stand for the average depths of ink penetration.

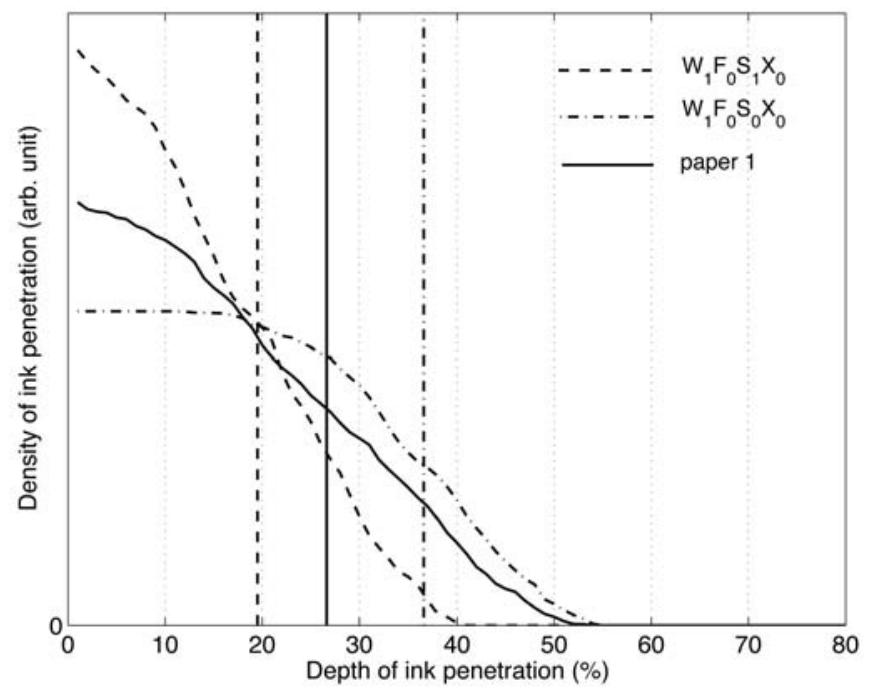

Fig 6. The distribution of ink density with respect to depth of ink-penetration. The samples are printed with cyan (ink level 3 ) on hand-sheets $W_{1} F_{0} S_{0} X_{0}, W_{1} F_{0} S_{1} X_{0}$, and office paper (paper sample 1). The plots have been normalized in order that all the samples have the same amount of ink. The vertical lines stand for the average depths of ink penetration.

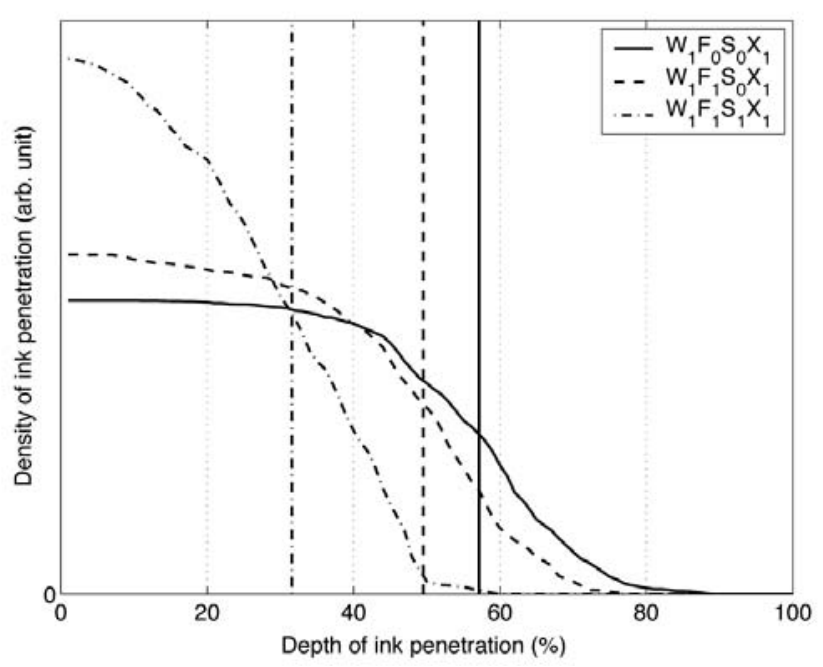

Fig 7. The distribution of ink density with respect to depth of ink-penetration. The samples were printed with cyan and yellow (ink level 3) on hand-sheets $W_{1} F_{0} S_{0} X_{1}, W_{1} F_{1} S_{0} X_{1}$, and $W_{1} F_{1} S_{1} X_{1}$. The plots have been normalized in order that all the samples have the same amount of ink. The vertical lines stand for the average depth of ink penetration.

average depth of ink penetration is denoted by a vertical line for each type of paper substrate. For $\mathrm{W}_{1} \mathrm{~F}_{0} \mathrm{~S}_{0} \mathrm{X}_{0}$, the dominant depth of ink penetration lies in a range between 30 to $50 \%$ of the paper's thickness, with the average depth of about $37 \%$. For $\mathrm{W}_{1} \mathrm{~F}_{0} \mathrm{~S}_{1} \mathrm{X}_{0}$ this interval is shifted downwards to between 10 to $35 \%$ due to the hydrophobizing effect. Correspondingly, the average depth of ink penetration (about 20\%) is around half that of $\mathrm{W}_{1} \mathrm{~F}_{0} \mathrm{~S}_{0} \mathrm{X}_{0}$. For the commercial sample 1, its histogram distribution lies in between those of the two hand-sheets.

The form of ink penetration may be even better described by the distribution of ink density in paper substrates, as shown in Fig 6. Since the samples were printed with the same ink level (3), they contain the same amount of ink. Therefore, all the plots have been normalized to the same amount of ink, i.e., the integrated areas under the density curves are equal. As paper $\mathrm{W}_{1} \mathrm{~F}_{0} \mathrm{~S}_{0} \mathrm{X}_{0}$ has the largest average depth of ink penetration, there is less ink concentration in the layer close to the substrate surface compared to the other two types of substrate. The ink distribution is practically homogeneous within a depth up to $19 \%$ and then decreases with increasing depth of penetration. The commercial sample 1, on the other hand, holds somewhat more ink on its surface and has an inhomogeneous ink distribution with respect to an increasing penetration depth. Paper $\mathrm{W}_{1} \mathrm{~F}_{0} \mathrm{~S}_{1} \mathrm{X}_{0}$ has the largest ink concentration near the surface and an even sharper density variation with depth. Thus internal sizing, especially in the absence of filler, hinders imbibition in the thickness direction. This observation reveals limitations of the theoretical model (Bristow 1987, Pauler 1987) in which homogeneous ink distribution within a certain depth of ink penetration is assumed.

Fig 7 depicts the density distribution of another three types of hand-sheets, without filler and size $\left(\mathrm{W}_{1} \mathrm{~F}_{0} \mathrm{~S}_{0} \mathrm{X}_{1}\right)$, with filler $\left(\mathrm{W}_{1} \mathrm{~F}_{1} \mathrm{~S}_{0} \mathrm{X}_{1}\right)$, and with both filler and size $\left(\mathrm{W}_{1} \mathrm{~F}_{1} \mathrm{~S}_{1} \mathrm{X}_{1}\right)$. As previously observed the sized paper holds much more dye on or close to the paper surface. On the other hand, as green is achieved by mixing drops of cyan with yellow ink, the prints contain almost double the amount of ink compared to primary colours at the same printing ink level, resulting in heavier ink penetration. Consequently, hand-sheets $\mathrm{W}_{1} \mathrm{~F}_{0} \mathrm{~S}_{0} \mathrm{X}_{1}$ and $\mathrm{W}_{1} \mathrm{~F}_{1} \mathrm{~S}_{0} \mathrm{X}_{1}$ have approximately uniform ink distributions to a large extent (up to a depth of about 40\%). The ink distribution in $\mathrm{W}_{1} \mathrm{~F}_{1} \mathrm{~S}_{1} \mathrm{X}_{1}$ containing internal size, however, is still far from uniform. Finally, visual inspection of Fig 4 reveals that cyan is well mixed with yellow as desired to produce the colour green.

Dependence of ink distribution on printed amount is another important perspective of ink penetration. Fig 8 depicts the density of magenta ink at printing level 1, 3, and 5 , on office copy paper (commercial sample 2). To make quantitative comparisons, the computed density values have been normalized to their respective ink amounts, with the integrated area under each density curve equalling 1,3 , and 5 times the basic drop amount. As shown, the prints of ink level 3 and 5 have almost identical ink density near their top surfaces, while the print of level 1 has much higher ink concentration near 


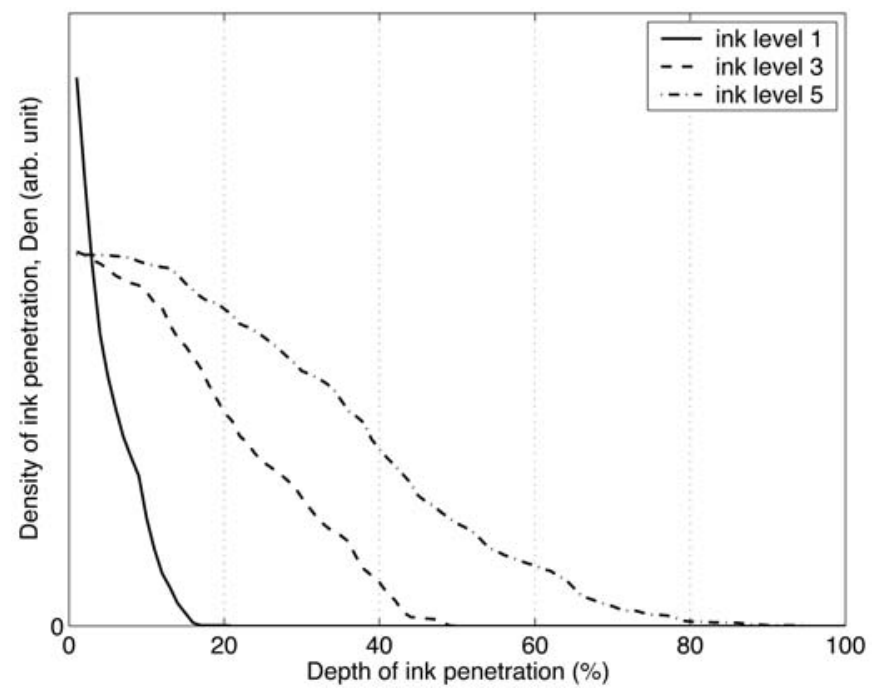

Fig 8. The distribution of ink density with respect to the amount of ink printed. The sam ples are printed with magenta on office paper, (paper sample 2). All the plots have been normalized to their respective ink amounts.

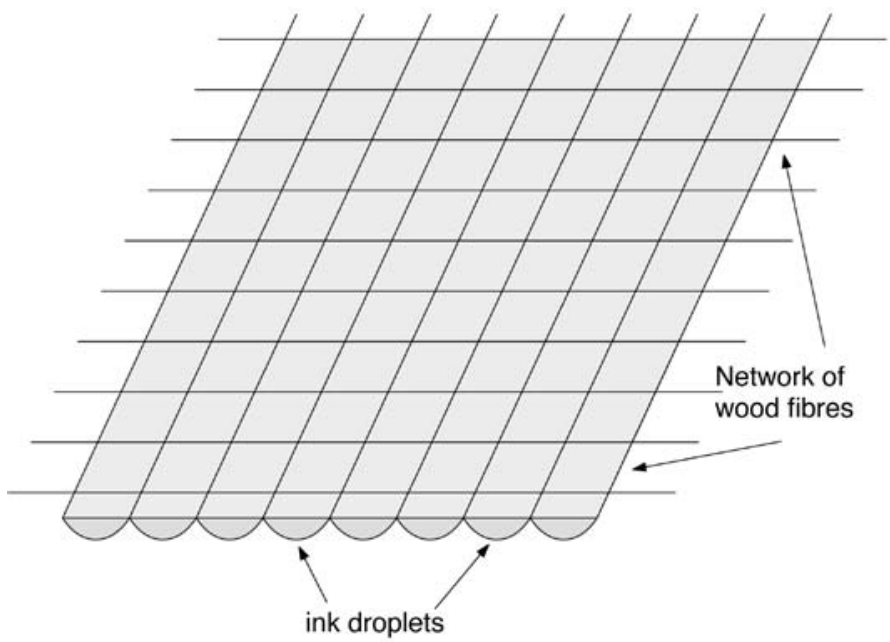

Fig 9. The schematic diagram for interaction between ink droplets and the network formed by wood fibres

its top surface. Analysis of prints of primary colours on other types of paper has confirmed these observations as general phenomena. A possible explanation relates to different mechanisms of ink-paper interaction. As depicted in Fig. 9 pulp fibres form networks, with which the ink droplets interact. At the lowest ink level 1, the interaction of ink with paper is of the form of individual separate drops spreading on the "dry" paper surface and penetrating into the surface pores. Accordingly the spreading and penetration proceeds by advancement of the threephase line on the "fresh" paper surfaces, which are hydrophobic (if internal size and/or surface size is present) and rough. The movement of the fluid at this threephase line is thus hindered by its relatively high contact angle on these low energy surfaces, together with the risk for its pinning due to roughness. For these reasons evaporation can proceed relatively fast compared to drop flow and immobilization of dye occurs close to the top surface. In contrast, at higher ink amounts multiple inkjet drops land on or close to each other in rapid succession. In this case subsequent drops land on the paper surface already primed by the drop first landing, and can thus

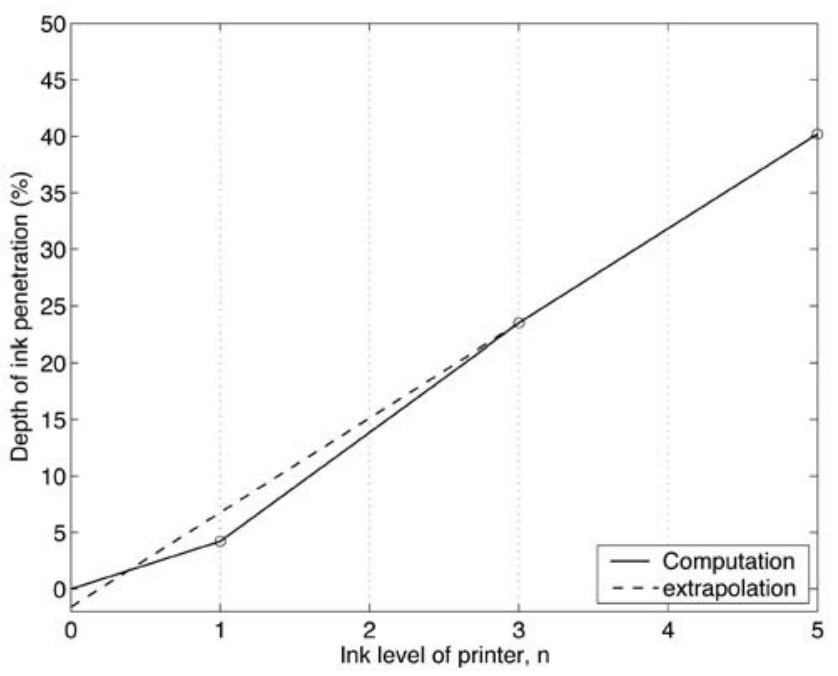

Fig 10. The variation in average depth of ink penetration for samples with printed (magenta) ink level 1, 3, and 5, respectively, on office paper (paper sample 2). The computed average depth based on image analysis is denoted by solid line and the extrapolated plot from ink level 3 and 5 by dashed line.

flow relatively easily over this liquid film rather than the hydrophobic and rough surface of the fresh paper. This establishes a renewing film flow enabling the fluid to penetrate deeper into the paper bulk, rather than being stranded close to the surface by lack of volume required to bridge over to the next fiber and continue flow. These effects combine to speed the rate of penetration relative to evaporation, thus dragging the ink deeper into the paper.

These phenomena are also responsible for the irregularity of the average depth of ink penetration, computed from $E q$ (3), with decreasing printed ink amount. Intuitively, one would expect the depth of ink penetration to vary linearly with ink amount, as suggested by the extrapolation (dashed line in Fig 10) based on the depths for ink levels 3 and 5. The extrapolation points to a vanished ink penetration when the printed ink amount becomes zero, while the average depth computed based on data from the image analysis (see Fig 10) deviate significantly from the linearity.

\section{Conclusions}

The method of combining microscopy with image analysis, to study ink-paper interaction or ink penetration, has been proposed and tested. Applications to prints on substrates of different material compositions and with different ink amounts demonstrate that the method is a powerful tool, with which characteristics of ink penetration can be described in a great detail. It is found that adding filler (calcium carbonate) has limited effects on reducing ink penetration even though it increases significantly the opacity of paper. Adding internal sizing (hydrophobizing) chemicals, however, can significantly reduce the ink penetration. For the same type of substrate, the depth of penetration increases linearly with increasing ink amount for sufficiently large amounts (ink level 3 or higher). However at lower ink amounts, a disproportionally high fraction of the ink is present at the paper surface (if hydrophobic), interpreted as being due to inability of 
these low amounts of ink to establish film flow over the low energy surfaces and access the paper's bulk. Therefore, the characteristics of ink penetration in substrates depend on ink surface tension, and bulk as well as surface properties of the paper sheet. The present study has contributed useful information about ink distribution in substrates, which is of great importance for modelbuilding and simulating the optical effects of ink penetration as outlined previously (Yang, Kruse 2000, Yang et al 2004). In general, imaging and analyzing fibre material for internal structure, formation (distribution), and defects has been of great interest in industrial research for the past ten years or more. Examples apart from inkpenetration are: fibre and pore structure and distribution in paper, original and replacement fibrous materials in the human body, the quality of standing trees and timber for the wood industry, etc. Although in this report we concentrated on investigating ink-jet ink distribution within a paper substrate, the results are relevant for a much wider set of applications.

\section{Acknowledgement}

This work has been financially supported by Swedish Foundation for Strategic Research through the Surface Science Printing Program (S2P2). One of the authors (LY) thanks Mr. Fayyazi at Tetra Pak (Sweden) for valuable discussions.

\section{Literature}

Bristow, J.A. (1987): Print-through and ink penetration - a mathematical treatment, Adv. Printing Sci. Techno. 19:137-145.

Emmel, P. and Hersch, R.D. (2002): Modelling ink spreading for colour prediction, J. Imaging Sci. Techno. 46:237-246.

Fayyazi, A. (2002): Texture based duplex-board layer segmentation, Licentiate thesis, LIU-TEK-LIC No. 932, Linköping University, Sweden.

Kishida, T., Fukui, T., Azuma, K., and Kanou, S. (2001): Influence of coating pore structure and ink set property on ink dry back in sheet-fed offset printing, Proc. 2001 Tappi Coating and Graphic Arts Conf. and Trade Fair, May 6-9 2001, San Diego, CA, pp.213-229.
Kubelka, P. and Munk, F. (1931): Ein Beitrag zur Optik der Farbanstriche, Z. Tech. Phys. (Leipzig), 12:593-601.

Kubelka, P. (1954): New contribution to the optics of intensely light-scattering materials. Part II, J. Opt. Soc. Am. 44:330-335.

McKnight, M.E., Vorburger, T.T., Marx, E., Nadal, M.E., Barnes, P.Y., and Galler, M.A. (2001): Measurements and predictions of light scattering by clean coatings, Appl. Opt. 40:2159-2168.

Mizutani, N. and Kondo, M. (2000): Ink absorption mechanism of silica based ink jet paper coating, Proc. IS\&T NIP16 Conf. pp.221-224.

Oittnen, P. (1976): Fundamental rheological properties and tack of printing inks and their influence on ink behaviour in a printing nip, PhD Dissertation, Helsinki University of Technology, Helsinki, Finland.

Pauler, N. (1987): A model for the interaction between ink and paper, Adv Printing Sci. and Techn. 19:116-136.

Pauler, N., Wågberg, J., and Eidenvall, L. (2002): Methods to determine ink penetration and light absorption of inkjet inks", Proc. International Printing \& Graphic Arts Conference (IPGAC), pp.33-36

Rousu, S. (2002): Differential absorption of offset ink constituents on coated paper, PhD thesis, Lab. of Paper Chem., Åbo AkademiUniversity, Finland.

Ström, G. (1988): Wetting studies related to offset printing, PhD Dissertation, Royal Institute of Technology, Stockholm, Sweden.

Ström, G., Gustafsson, J., and Sjölin, K. (2000): Separation of ink constituents during ink setting on coated substrates, Proc. Printing and Graphic Arts Conf., pp. 89-99.

Sung, L.P., Nadal, M.E., McKnight, M.E., Marx, E., and Laurent, B. (2002): Optical reflectance of metallic coatings: Effect of aluminium flake orientation, J. Coatings Techn. 74:55-63.

Voet, A. (1952): Ink and paper in the printing process, Inter Science Publishers Inc., New York.

Vyörykkä, J. (2004): Confocal Raman Microscopy in Chemical and Physical Characterization of Coated and Printed Papers, PhD Thesis, Helsinki University of Technology, Finland.

Yang, L. and Kruse, B. (2000): Ink penetration and its effect on printing, Proc. SPIE (The International Society for Optical Engineering), 3963:365-375

Yang, L., Kruse, B, and Miklavcic, S.J. (2004): Revised Kubelka-Munk theory. II. A study of ink penetration in ink-jet printing, J. Opt. Soc. Am. A 21:1942-1952.

Manuscript received March 9, 2005 Accepted August, 2005 